\title{
Correlation between the Sample Mean and Sample Variance
}

Ramalingam Shanmugam

Texas State University, rs25@txstate.edu

Follow this and additional works at: http://digitalcommons.wayne.edu/jmasm

Part of the Applied Statistics Commons, Social and Behavioral Sciences Commons, and the Statistical Theory Commons

\section{Recommended Citation}

Shanmugam, Ramalingam (2008) "Correlation between the Sample Mean and Sample Variance," Journal of Modern Applied Statistical Methods: Vol. 7 : Iss. 2 , Article 6.

DOI: $10.22237 /$ jmasm/1225512300

Available at: http://digitalcommons.wayne.edu/jmasm/vol7/iss2/6

This Regular Article is brought to you for free and open access by the Open Access Journals at DigitalCommons@WayneState. It has been accepted for inclusion in Journal of Modern Applied Statistical Methods by an authorized editor of DigitalCommons@WayneState. 


\title{
Correlation between the Sample Mean and Sample Variance
}

\author{
Ramalingam Shanmugam \\ Texas State University-San Marcos
}

This article obtains a general formula to find the correlation coefficient between the sample mean and variance. Several particular results for major non-normal distributions are extracted to help students in classroom, clients during statistical consulting service.

Key words: Skewness, kurtosis, non-normal data, count and continuous distributions.

\section{Introduction}

Interest about the relationship between the sample descriptive measures is growing among the statisticians. For example, Zhang (2007) using a lengthy combinatorial argument obtained an expression for computing the covariance of sample mean and sample variance without the assumption of normality to help teachers explain to students. Such a tedious combinatorial new derivation is obsolete as it is a direct consequence of the results in Stuart and Ord (1994). Their result is helpful to find additionally the covariance between the sample mean and any even moment about the mean. However, no formula appears for computing the correlation between the sample mean and variance of a non-normal sample. Yet, almost all students in statistics courses and the clients during statistical consulting service curiously seek to know an estimate of such correlation in their data. So, there is a need to have a list of expressions for non- normal data which the statisticians can readily use to answer the clients' query.

Ramalingam Shanmugam is a Professor in the School of Health Administration. His research interests include multivariate data modeling, informatics, optimal decision support systems, and health/medical application of statistics. Email: rs25@txstate.edu.
Let $\bar{X}=n^{-1} \sum_{i=1}^{n} X_{i}=m_{1}^{\prime}$ and $\left(\frac{n}{n-1}\right) m_{2}=$ $S^{2}=(n-1)^{-1} \sum_{i=1}^{n}\left(X_{i}-\bar{X}\right)^{2}$ be the sample mean and variance of a random sample drawn from a given population, where $m_{1}$ and $m_{2}$ are the notations in Stuart and Ord (1994, volume 1, page 350). A consequence of their results is that

$$
\operatorname{Cov}\left[\bar{X}, S^{2}\right]=\operatorname{Cov}\left[m_{1}^{\prime}, m_{2}\right)=\frac{\mu_{3}}{n} .
$$

\section{Methodology}

In the next two sections, the general formula for finding the correlation and results for particular specified non-normal samples are obtained. The results for Poisson, geometric, and Bernoulli samples are illustrated with data from the literature for better understanding.

Derivation of formula for $\operatorname{Corr}\left(\bar{X}, S^{2}\right)$

Because $\operatorname{Var}[\bar{X}]=\operatorname{Var}\left[m_{1}^{\prime}\right]=\frac{\sigma^{2}}{n}$

and

$$
\begin{aligned}
& \operatorname{Var}\left[S^{2}\right]=\operatorname{Var}\left[\left(\frac{n}{n-1}\right) m_{2}\right] \\
& =\frac{\mu_{4}-\left(\sigma^{2}\right)^{2}}{n}+\frac{2\left(\sigma^{2}\right)^{2}}{n(n-1)}
\end{aligned}
$$

due to (10.9) in Stuart and Ord (1994) where $\sigma^{2}$ is the population variance. The kurtosis is measure of tail flatness of the frequency trend of 
the data. Because the kurtosis is defined to be

$$
\begin{aligned}
& K_{u}=\frac{\mu_{4}}{\left(\sigma^{2}\right)^{2}}, \text { rewrite } \\
& \quad \operatorname{Var}\left[S^{2}\right]=\frac{\left(\sigma^{2}\right)^{2}}{n}\left[K_{u}-1+\frac{2}{(n-1)}\right]
\end{aligned}
$$

Similarly, the skewness is a measure of the lack of symmetry in the frequency trend of the data. The skewness is defined as $S_{k}=\frac{\mu_{3}^{2}}{\left(\sigma^{2}\right)^{3}}$. Using (1) through (3), the correlation coefficient between the sample mean and sample variance is obtained and after algebraic simplifications, it is

$$
\operatorname{Corr}\left[\bar{X}, S^{2}\right]=\sqrt{\left|\frac{S_{k}}{K_{u}-1+\frac{2}{n-1}}\right|}, n \geq 2
$$

With only one observation (that is, $\mathrm{n}=$ 1), the correlation between the sample mean and variance cannot be determined if the skewness is non zero, according to (4) as it requires multiple observations. Also, from expression (4), notice that the correlation is zero when the skewness is zero and it occurs in a random sample from a symmetric population. The $t$, Laplace, error distribution, the discrete and continuous uniform probability distributions in addition to normal distribution are symmetric population with zero skewness. Hence, the zero correlation between the sample mean and sample variance does not necessarily mean only the normal population due to (4). Furthermore, notice in (4) that the correlation weakens as the sample size increases. The skewness and kurtosis moderate the correlation coefficient in a way. The details are discussed, listed and illustrated below in several cases. In the next section, the results for particular non-normal cases which are come across in graduate courses and statistical consulting service.

Special non-normal cases

The power of mathematical statistics enables to group several probability distributions under one "umbrella" as they possess a common property.
(Modified) power series family sample

One such property is power series nature of the probability mass function (pmf). The pmf of power series distribution is defined (it seems earliest by Kosambi, 1949) to be

$$
\operatorname{Pr}[x]=\frac{a_{x} \theta^{x}}{\eta(\theta)}
$$

with a non-negative and differentiable function $\eta(\theta)$ of a natural parameter $\theta$. The variance in this family is

$$
\sigma^{2}=\theta^{2} \partial_{\theta \theta}^{2} \ln \eta(\theta)+\theta \partial_{\theta} \ln \eta(\theta)
$$

where $\partial_{\theta \theta}^{k}$ means the $\mathrm{k}$-th derivative with respect to the natural parameter. The skewness is

$$
S_{k}=\left(\theta \partial_{\theta} \sigma^{2}\right)^{2} / \sigma^{6}
$$

The kurtosis is

$$
K_{u}=\left(\theta \partial_{\theta} \mu_{3}+3 \sigma^{4}\right) / \sigma^{4}
$$

Substituting (6), (7), and (8) in (4), the correlation for the power series family could be readily computed.

This family is modified in several ways. One modification is by Gupta (1974), who introduced a modified power series distribution (MPSD) with pmf

$$
\operatorname{Pr}[x]=\frac{a_{x}[u(\theta)]^{x}}{\eta(\theta)}
$$

The variance, skewness, and kurtosis in (6), (7), and (8) change to

$$
\begin{gathered}
\sigma^{2}=\frac{\partial_{\theta}\left[\frac{\partial_{\theta} \ln \eta(\theta)}{\partial_{\theta} \ln u(\theta)}\right]}{\partial_{\theta} \ln u(\theta)}, \\
S_{k}=\left(\frac{\partial_{\theta} \sigma^{2}}{\partial_{\theta} \ln u(\theta)}\right)^{2} / \sigma^{6},
\end{gathered}
$$

and

$$
K_{u}=\left(\partial_{\theta}\left[\frac{\partial_{\theta} \ln \eta(\theta)}{\partial_{\theta} \ln u(\theta)}\right]+3 \sigma^{4}\right) / \sigma^{4} .
$$

for MPSD. By substituting in (4), the correlation for the modified power series family can be computed. 


\section{CORRELATION BETWEEN THE SAMPLE MEAN AND SAMPLE VARIANCE}

Binomial sample (with replacement)

For binomial sample, one need to consider $\eta(\theta)=(1+\theta)^{r}$ with $r \geq 1$ denotes the number of trials and the natural parameter $\theta=p /(1-p)$. By substituting the skewness

$$
\hat{S}_{k}=\left(1-\frac{2 \bar{x}}{r}\right)^{2}\left(\bar{x}\left[1-\frac{\bar{x}}{r}\right]\right)^{-1}
$$

and the kurtosis

$$
\hat{K}_{u}=3+\left(1-\frac{6 \bar{x}}{r}\left[1-\frac{\bar{x}}{r}\right]\right)\left(\bar{x}\left[1-\frac{\bar{x}}{r}\right]\right)^{-1},
$$

in (4), the correlation of the binomial sample mean and variance is noticed. When the number of trials is large (that is, $r \rightarrow \infty$ ), the correlation diminishes but not to zero.

\section{Bernoulli trials}

With $r=1$ in the above binomial results, note that the correlation for Bernoulli sample mean and variance is

$$
\begin{aligned}
& \operatorname{Corr}\left[\bar{X}, S^{2}\right] \\
& =(1-2 \bar{x})\left[1+\left(\frac{2 n}{n-1}-6\right) \bar{x}(1-\bar{x})\right]^{-1}
\end{aligned}
$$

This is useful in discussions of the logistic regression data. Consider the following partial data (Dalal, et al., 1989) of $n=5$ observations with respect to failure $(\mathrm{X}=1)$ and non failure $(\mathrm{X}$ $=0)$ of O-rings in space rockets. The shuttle challenger exploded after its launch on 28 January 1986 with a loss of seven lives. A commission was charged with determining the causes of that tragedy. Their report concluded that the failure of O-rings in nozzle joints due to thermal stress was the reason. The gas went through the cracks in the stressed O-rings caused the explosion.

\section{Poisson sample}

The Poisson distribution is a limiting case of binomial distribution when the Bernoulli chance $\mathrm{p}$ is small but the number of trials is large enough to make a finite mean $\theta$. If the number of O-rings to be investigated is large and the chance of any failure is very slim, then the expected number of O-ring failures is $\theta>0$ and it is the mean of Poisson frequency trend. For such a Poisson sample, note that $\eta(\theta)=e^{\theta}$ with
Table 1. Date and O-ring failure $(\mathrm{X}=1)$ or non-failure $(X=0)$ of $n=5$ cases

\begin{tabular}{|c|c|c|c|c|c|}
\hline Date & $\begin{array}{c}21 \\
\text { April } \\
81\end{array}$ & $\begin{array}{c}12 \\
\text { Nov } \\
1981\end{array}$ & $\begin{array}{c}8 \\
\text { Nov } \\
1984\end{array}$ & $\begin{array}{c}30 \\
\text { Aug }\end{array}$ & $\begin{array}{c}21 \\
\text { Jan } \\
1984\end{array}$ \\
\hline $\mathrm{X}=$ & 0 & 1 & 0 & 1 & 1 \\
\hline
\end{tabular}

Note: the sample mean $\bar{x}=0.6$ and sample variance $s^{2}=0.24$ with $\mathrm{n}=$ 5. Substituting in (9), the correlation coefficient between the Bernoulli sample mean and sample variance is computed and it is $\operatorname{Corr}\left[\bar{X}, S^{2}\right]=0.489$.

the natural parameter $\theta$ denoting the incidence rate in the power series family. Substituting the Poisson skewness and kurtosis

$$
S_{k}=\theta^{-1}=K_{u}-3
$$

in (4), the correlation of the Poisson sample mean and variance could be obtained. It is

$$
\begin{aligned}
& \operatorname{Corr}\left[\bar{X}, S^{2}\right] \\
& =\left[2\left(\hat{\theta}+\frac{1}{n-1}\right)-1\right]^{-1 / 2} . \\
& =\left[2\left(\bar{x}+\frac{1}{n-1}\right)-1\right]^{-1 / 2}
\end{aligned}
$$

With the larger incidence rate, the Poisson correlation diminishes.

Incidence rate restricted Poisson sample

In spite of rarity in the Poisson data, sometimes the data might not be well governed by the above described Poisson distribution. A modification in the Poisson probability distribution is necessary. One such modification is due to Shanmugam's (1991). When the regular Poisson distribution does not fit a given data, one could consider the incidence rate restricted Poisson distribution (IRRPD) because it is versatile enough to fit the data. The pmf of IRRPD is

$$
\operatorname{Pr}[x]=\frac{(1+\gamma x)^{x-1}\left(\theta e^{-\gamma \theta}\right)^{x}}{x ! e^{\theta}}
$$

where the incidence $\operatorname{rate} \theta \leq \frac{1}{\gamma}$ and $\gamma$ is the restriction parameter. The restriction is removed when $\gamma$ approaches zero and in which case, it reduces to the Poisson distribution in section (3.4). The skewness and kurtosis are 


$$
S_{k}=(1+2 \gamma \theta)^{2}[\theta(1-\gamma \theta)]^{-1}
$$

and

$$
K_{u}=3+\left(1+8 \gamma \theta+6 \gamma^{2} \theta^{2}\right)[\theta(1-\gamma \theta)]^{-1}
$$

The estimates of the IRRPD parameters are $\hat{\gamma} \hat{\theta}=1-\sqrt{\frac{\bar{x}}{s^{2}}} \quad$ and $\hat{\theta}=\bar{x} \sqrt{\frac{\bar{x}}{s^{2}}} . \quad$ Substituting these estimates in (4), the correlation of the Poisson sample mean and variance can be obtained. When $\gamma$ approaches zero, the above results reduce to those in Section 3.4 for the Poisson distribution.

To illustrate, consider the following data in the literature about the number of tram accidents, X in Belgrade during 1965 and 1970 from Shanmugam and Singh (2001) as redisplayed in the Table 2 below. The estimate of the IRRPD parameters with data on $\mathrm{n}=134$ drivers are $\hat{\theta}=3.724$ and $\hat{\gamma}=0.101$.

Table 2. \# Tram Accidents in Belgrade

\begin{tabular}{|l|c|c|c|c|c|c|c|c|c|c|c|}
\hline $\mathrm{X}=$ & 0 & 1 & 2 & 3 & 4 & 5 & 6 & 7 & 8 & 9 & $\begin{array}{c}\geq \\
10\end{array}$ \\
\hline $\mathrm{f}=$ & 1 & 8 & 14 & 17 & 16 & 19 & 16 & 9 & 6 & 6 & 21 \\
\hline
\end{tabular}

With these estimates, the skewness, kurtosis, and hence the correlation between the sample mean and variance are obtained and they are $\hat{S}_{k}=1.321, \hat{K}_{u}=5.089$ and

$$
\operatorname{Corr}\left[\bar{X}, S^{2}\right]=0.567 \text {, respectively. }
$$

For another example, consider the number of injury accidents that occurred in the Interstate-95 during January 1, 1969 through October 31, 1970 as reported in Shanmugam and Singh (1981).

Table 3. \# Injury Accidents in Virginia State during January 1, 1969 \& October 31, 1970

\begin{tabular}{|c|c|c|c|c|c|c|}
\hline $\mathrm{X}=$ & 0 & 1 & 2 & 3 & 4 & $5+$ \\
\hline $\mathrm{f}=$ & 286 & 216 & 92 & 30 & 14 & 1 \\
\hline
\end{tabular}

With $\mathrm{n}=639$, the estimates of IRRPD are $\hat{\theta}=0.06$ and $\hat{\gamma}=13.5$. Hence, the skewness, kurtosis, and the correlation between the IRRPD sample mean and variance are

$$
\hat{S}_{k}=287.3, \hat{K}_{u}=1003.403
$$

and

$$
\operatorname{Corr}\left[\bar{X}, S^{2}\right]=0.535,
$$

respectively.

Inverse binomial sample

With $\eta(\theta)=(1-\theta)^{-r}$ in the power series family, $r \geq 1$ denoting the number of cases to be of a particular, and the natural parameter $\theta=p$ be the probability of outcome of a type, the number of cases to be investigated is an inverse binomial random variable. Substituting its skewness

$$
\hat{S}_{k}=(2-\hat{p})^{2}(r[1-\hat{p}])^{-1}
$$

and the kurtosis

$$
\hat{K}_{u}=3+\frac{\hat{p}^{2}+6[1-\hat{p}]}{r[1-\hat{p}]}
$$

with $\hat{p}=\frac{\bar{x}-1}{r+\bar{x}-1}$. Substituting in (4), the correlation of the inverse binomial sample mean and variance is obtainable.

Geometric sample

With $r=1$ in the above results for the inverse binomial sample, the correlation between the geometric mean and sample variance of a geometric sample is obtained and it is

$$
\operatorname{Corr}\left[\bar{X}, S^{2}\right]=\frac{\bar{x}+1}{\sqrt{\bar{x}\left(7 \bar{x}+\bar{x}^{-1}+2[n-1]^{-1}\right)}} \cdot
$$

For illustration, consider the geometric sample data on the number of heart failures experienced by a random sample of $n=15$ cardiology patients in Alabama state as used in Bartolucci et al (1999). The sample mean is 1.2 in that geometric data. Substituting in (11) the sample mean, the correlation between the sample mean and variance of the geometric data is obtained and it is $\operatorname{Corr}\left[\bar{X}, S^{2}\right]=0.657$.

Log series sample

For sample from a logarithmic series distribution, note that $\eta(\theta)=-\ln (1-\theta)$ with the natural parameter $\theta$ in power series family. Substituting its skewness 


$$
S_{k}=\frac{\left[(1+\theta)+\frac{3 \theta}{\ln (1-\theta)}+\frac{2 \theta^{2}}{(\ln (1-\theta))^{2}}\right]^{2}}{\left[\frac{\theta}{\ln (1-\theta)}\right]^{2}\left[1+\frac{\theta}{\ln (1-\theta)}\right]^{3}}
$$

and the kurtosis

$$
\begin{gathered}
{\left[1+4 \theta+\theta^{2}+\frac{4 \theta(1+\theta)}{\ln (1-\theta)}+\right.} \\
K_{u}=\frac{\left.\frac{6 \theta^{2}}{(\ln (1-\theta))^{2}}+3 \frac{\theta^{3}}{(\ln (1-\theta))^{3}}\right]}{\left[\frac{-\theta}{\ln (1-\theta)}\right]\left[1+\frac{\theta}{\ln (1-\theta)}\right]^{2}}
\end{gathered}
$$

in (4), the correlation between the binomial sample mean and variance could be obtained.

Hypergeometric sample (without replacement)

In many health applications, random sampling is done without replacement. For an example, once the virus infected individuals are identified in the population and are kept removed from the community. Suppose that $N p$ individuals are suspected to be infected where $0<p<1$ indicates the prevalence level of the virus in the community. The number of infected individuals in a random sample of $\mathrm{n}$ persons without replacement is a hypergeometric outcome. Its skewness and kurtosis in data of this type are respectively

$$
S_{k}=\frac{\left(1-\frac{1}{N}\right)}{n p(1-p)\left(1-\frac{n}{N}\right)}\left[\frac{(1-2 p)\left(1-\frac{2 n}{N}\right)}{\left(1-\frac{2}{N}\right)}\right]^{2}
$$

and

$$
\begin{aligned}
& {[(N-1)[N(N+1-6 n)} \\
& +3 p(1-p)(n-2)+6(n / N)^{2} \\
& +3 p(1-p)(n / N)(6-n) \\
K_{u}=3+ & \frac{\left.-18 p(1-p)(n / N)^{2}\right]}{n p(N-n)(N-2)(N-3)}
\end{aligned}
$$

Substituting in (4), the correlation between the hypergeometric sample mean and variance could be obtained.
Katz's family sample

The pmf of the Katz's family is denoted by $\operatorname{Pr}[x+1]=\left(\frac{\alpha+\beta x}{1+x}\right) \operatorname{Pr}[x]$. Its skewness and kurtosis are

$$
S_{k}=\left(\frac{2}{\beta}-1\right) / \sigma^{2}
$$

and

$$
K_{u}=3+\left(\frac{6}{(1-\beta)^{2}}-\frac{6}{(1-\beta)}+1\right) / \sigma^{2}
$$

respectively. Substituting in (4), the correlation between the sample mean and variance of Katz's family can be obtained.

Log-normal sample

Consider a random sample is drawn from a log-normal population with the threshold, location, and scale parameters $\theta, \xi, \sigma^{2}$ respectively. The skewness and kurtosis are

$$
S_{k}=(\varpi+2)^{2}(\varpi-1)
$$

and

$$
K_{u}=\varpi^{4}+2 \varpi^{3}+3 \varpi^{2}-3
$$

with $\varpi=e^{\sigma^{2}}$. Substituting in (4), the correlation between the log-normal sample mean and variance could be obtained.

Gamma sample

The skewness and kurtosis of the gamma population with pdf $f(x)=\left(\frac{\mu}{\sigma^{2}}\right)\left(\frac{x \mu}{\sigma^{2}}\right)^{\left(\frac{\mu}{\sigma}\right)^{2}-1} e^{-\left(\frac{x \mu}{\sigma^{2}}\right)} / \Gamma\left(\left(\frac{\mu}{\sigma}\right)^{2}\right)$

are $S_{k}=4\left(\frac{\mu}{\sigma}\right)^{-2}$ and $K_{u}=3+6\left(\frac{\mu}{\sigma}\right)^{-2}$ where $\mu$ and $\sigma^{2}$ denote the mean and variance. Substituting in (4), the correlation between the gamma sample mean and variance is obtained. In the gamma case, it is

$\operatorname{Corr}\left[\bar{X}, S^{2}\right]=\sqrt{\frac{2 \sigma^{2}}{\mu^{2}+3 \sigma^{2}+[n-1]^{-1}}}$. 
Exponential sample

When $\mu=\sigma$ in the above results, they reduce to those for exponential population. The exponential population is an interesting special case. Then, the correlation between the exponential sample mean and variance is

$$
\operatorname{Corr}\left[\bar{X}, S^{2}\right]=\sqrt{\frac{2 \bar{x}^{2}}{4 \bar{x}^{2}+[n-1]^{-1}}}
$$

For an illustration, consider Zelen's exponential data below about the number of weeks a random sample of $\mathrm{n}=11$ tumor patients survived in a health clinic. The data are well fit by an exponential distribution as it is verified in Shanmugam (1991).

Table 4. Zelen's data of survival weeks of $n=$ 11 tumor patients

\begin{tabular}{|l|l|l|l|l|l|l|l|l|l|l|}
\hline 3 & 4 & 5 & 8 & 8 & 10 & 12 & 16 & 17 & 30 & 33 \\
\hline
\end{tabular}

The sample mean is equal to 13.6 weeks. According to (12), the correlation between the exponential sample mean and variance of this exponential data is $\operatorname{Corr}\left[\bar{X}, S^{2}\right]=0.71$.

Inverse gaussian sample

The inverse Gaussian distribution is considered as an alternate model for positive but skewed data. Its skewness and kurtosis are

$$
\hat{S}_{k}=\frac{9 \bar{x}\left[\sum_{i=1}^{n} x_{i}^{-1}-(\bar{x})^{-1}\right]}{n-1}
$$

and

$$
\hat{K}_{u}=3+\frac{15 \bar{x}\left[\sum_{i=1}^{n} x_{i}^{-1}-(\bar{x})^{-1}\right]}{n-1}
$$

respectively. Substituting in (4), the correlation between the sample mean and variance of inverse Gaussian data can be obtained.

\section{Pareto sample}

The Pareto distribution is considered another alternate model for positive but skewed data. Its skewness and kurtosis are

$$
\hat{S}_{k}=4\left[\frac{\left(\sqrt{1+\left(\frac{\bar{x}}{s}\right)^{2}}+2\right)^{2}\left(\sqrt{1+\left(\frac{\bar{x}}{s}\right)^{2}}-1\right)}{\left(\sqrt{1+\left(\frac{\bar{x}}{s}\right)^{2}}-2\right)^{2}\left(\sqrt{1+\left(\frac{\bar{x}}{s}\right)^{2}}+1\right)}\right]
$$

and

$$
\hat{K}_{u}=\frac{3\left(\sqrt{1+\left(\frac{\bar{x}}{s}\right)^{2}}-1\right)\left[3\left(\sqrt{1+\left(\frac{\bar{x}}{s}\right)^{2}}+1\right)^{2}+\sqrt{1+\left(\frac{\bar{x}}{s}\right)^{2}}-2\right]}{\left(\sqrt{1+\left(\frac{\bar{x}}{s}\right)^{2}}+1\right)\left(\sqrt{1+\left(\frac{\bar{x}}{s}\right)^{2}}-2\right)\left(\sqrt{1+\left(\frac{\bar{x}}{s}\right)^{2}}-3\right)}
$$

respectively. Substituting in (4), the correlation between the sample mean and variance of Pareto data can be obtained.

Beta sample

The beta distribution is considered suitable for percentage data. Its skewness and kurtosis are

$$
\left.\hat{S}_{k}=\frac{4(\hat{\oplus}-\hat{v})^{2}(\hat{\oplus}+\hat{v}+1)}{(\hat{\oplus}+\hat{v}+1)^{2} \hat{\oplus} \hat{v}}\right]
$$

and

$$
\hat{K}_{u}=\frac{3(\hat{\omega}+\hat{v})(\hat{\omega}+\hat{v}+1)(\hat{v}+1)(2 \hat{\omega}-\hat{v})}{\hat{\omega} \hat{v}(\hat{\omega}+\hat{v}+2)(\hat{\omega}+\hat{v}+3)}+\frac{\hat{v}(\hat{v}-\hat{\omega})}{(\hat{\omega}+\hat{v})}
$$

respectively. Substituting in (4), the correlation between the sample mean and variance of beta data can be obtained where $\hat{v}=\bar{x}\left\{\frac{\bar{x}(1-\bar{x})}{s^{2}}-1\right\}$ and

$$
\hat{v}=(1-\bar{x})\left\{\frac{\bar{x}(1-\bar{x})}{s^{2}}-1\right\} .
$$

(Non) central chi-squared sample

The non-central chi-squared sample is considered and analyzed in the discussion of statistical power calculation of hypothesis testing or analysis of variance. Its skewness and kurtosis are

$$
\hat{S}_{k}=\frac{64\left(s^{2}-\bar{x}\right)^{2}}{s^{6}}
$$

and 


$$
\hat{K}_{u}=3+\frac{24\left(3 s^{2}-4 \bar{x}\right)}{s^{4}}
$$

respectively. Substituting these in (4), the correlation between the sample mean and variance of non-central chi-squared observations can be obtained. When $s^{2}=2 \bar{x}$, all the above results reduce to those of central chi-squared sample.

\section{(Non) central F sample}

The non-central $\mathrm{F}$ sample is considered in the discussion of statistical power calculation of hypothesis testing or analysis of variance. The results are too messy to mention. However, the skewness and kurtosis of the central $\mathrm{F}$ sample are

$$
\hat{S}_{k}=\frac{8(2 \hat{v}+\hat{\varpi}-2)(\hat{\oplus}-4)}{\hat{v}(\hat{\varpi}-6)(\hat{v}+\hat{\varpi}-2)}
$$

and

$$
\begin{aligned}
& \hat{K}_{u}= \\
& 3+\frac{12\left[(\hat{\varpi}-2)^{2}(\hat{\varpi}-4)+\hat{v}(\hat{v}+\hat{\varpi}-2)(5 \hat{\varpi}-22)\right]}{\hat{v}(\hat{\oplus}-6)(\hat{\varpi}-8)(\hat{v}+\hat{\varpi}-2)}
\end{aligned}
$$

respectively, where

$$
\grave{\oplus}=\frac{2 \bar{x}}{\bar{x}-1}
$$

and

$$
\hat{v}=\frac{(\bar{x}-1)^{2} s^{2}(2-\bar{x})}{\bar{x}^{2}}-2
$$

Substituting these in (4), the correlation between the sample mean and variance of central $\mathrm{F}$ observations can be obtained.

(Non) central t sample

The non-central t sample is considered in the discussion of testing one sided hypothesis. Its skewness and kurtosis are

$$
\hat{S}_{k}=\hat{\delta}\left(3+1.25 \frac{\hat{\delta}^{2}}{\hat{v}}\right)^{2} / \hat{v}
$$

and

$$
\hat{K}_{u}=\frac{1.406(\hat{v}-3.2)}{(\hat{v}-4)} \beta_{1}+\frac{3(\hat{v}-2)}{(\hat{v}-4)}
$$

Where

$$
\hat{\delta}=\left(s^{2}+\bar{x}^{2}\right)\left(\frac{\hat{v}^{2}-2}{\hat{v}}-1\right)^{1 / 2}
$$

and

$$
\frac{\bar{x}}{\hat{\delta}}=\frac{\sqrt{\frac{\hat{v}^{2}}{2}} \Gamma\left(\frac{\hat{v}^{2}-1}{2}\right)}{\Gamma\left(\frac{\hat{v}^{2}}{2}\right)}
$$

Substituting these in (4), the correlation between the sample mean and variance of noncentral $t$ observations can be obtained.

Power function sample

In financial studies, random sample of observations is well fit by a power function distribution. The skewness and kurtosis in power function distribution are estimated using

$$
\hat{S}_{k}=\frac{4(1-\hat{c})^{2}(2+\hat{c})}{(3+\hat{c})^{2} \hat{c}}
$$

and

$$
\hat{K}_{u}=\frac{3(\hat{c}+2)\left[2(\hat{c}+1)^{2}+\hat{c}(\hat{c}+5)\right]}{\hat{c}(\hat{c}+3)(\hat{c}+4)}
$$

where $\hat{c}=\sqrt{1+\left(\frac{\bar{x}}{s}\right)^{2}}-1$.

Substituting the skewness and kurtosis in formula (4), the correlation between the sample mean and variance of the power function sample can be obtained.

\section{References}

Bartolucci, A., Shanmugam, R., \& Singh, K. (1999). Geometric Model Generalized for Cardiovascular Studies. Epidemiology, Health, and Medical Research, 2. L. Oxley, F. Scrimgeour, \& M. McAleer, (Eds.). Modeling and Simulation Society of Australia Inc., p. 501506.

Dalal, S. R., Fowkles, E. B., \& Hoadley, B. (1989). Risk analysis of the space shuttle: Pre-Challenger prediction of failures. Journal of the American Statistical Association, 84, 945957.

Gupta, R. C. Modified power series distributions and some of its applications, Sankhya, 35, 288-298. 


\section{SHANMUGAM}

Kosambi, D. D. (1949). Characteristic properties of series distributions. Proceedings of the National Institute of Science, India, 15, 109113.

Shanmugam, R., \& Singh, K. (2001). Testing of Poisson incidence rate restriction. International Journal of Reliability and Applications, 2, 263-268.

Shanmugam, R. (1991). Testing guaranteed exponentiality. Naval Research Logistics, 38, 877-892.
Shanmugam, R., \& Singh, J. (1981) Some bivariate probability models applicable to traffic accidents and fatalities data, Statistical Distributions in Scientific Work. C. Tallie, et al. (Eds.), 4, 95-103.

Stuart, A., \& Ord, J. K. (1994) Kendall's advanced theory of statistics, Volume 1. London: Arnold Publisher.

Zhang, L. (2007) Sample mean and sample variance: Their covariance and their (in)dependence. American Statistician, 61, 159160. 